Int. J. Electrochem. Sci., 14 (2019) $7961-7972$

\title{
Construction of Highly Active Pt/Ni-Fe Layered Double Hydroxide Electrocatalyst towards Methanol Oxidation in Alkaline Medium
}

\author{
Haiyan Wang ${ }^{1}$, Yanan Chen ${ }^{1}$, Wenfu Xie ${ }^{1}$, Xiaoxue Han ${ }^{1}$, Qian Feng ${ }^{1}$, Rong Jiang ${ }^{1}$, Hu Shang ${ }^{1}$, \\ Feifei Zhang ${ }^{*}$, , Linna Gao ${ }^{2}$, Zonghua Wang ${ }^{1}$ \\ ${ }^{1}$ College of Chemistry and Chemical Engineering, Shandong Sino-Japanese Center for \\ Collaborative Research of Carbon Nanomaterials, Qingdao University, Qingdao 266071, China \\ ${ }^{2}$ College of Chemical and Environmental Engineering, Shandong University of Science and \\ Technology, Qingdao, 266590, China \\ *E-mail: zhangfeifei00921@163.com
}

doi: $10.20964 / 2019.08 .06$

Received: 20 March 2019 / Accepted: 15 May 2019 / Published: 30 June 2019

\begin{abstract}
A novel Pt/Ni-Fe layered double hydroxide (Pt/Ni-Fe LDH) electrocatalyst was fabricated by a two-step facile process for oxidation of methanol in alkaline medium. It is found that $\mathrm{Pt} / \mathrm{Ni}-\mathrm{Fe} \mathrm{LDH}$ electrocatalyst exhibits higher electrocatalytic activity, better anti-poisoning ability and stability for methanol oxidation comparing to pure Pt black and commercial Pt/C catalyst. Such an enhancement is probably attributed to the synergistic effect of Ni-Fe LDH and the uniform loading of Pt nanoparticles on the LDH layers. When ionic liquid (IL) was introduced to the composites, the prepared Pt/IL/Ni-Fe LDH exhibits even better electrocatalytic behaviors $\left(205.6 \mathrm{~mA} \mathrm{mg}^{-1} \mathrm{Pt}, I_{f} / I_{b}=5.67\right)$ than the Pt/Ni-Fe LDH. Moreover, the morphology and structure of composites were characterized using scanning electron microscope equipped with an energy dispersive X-ray spectrometry, transmission electron microscope, $\mathrm{X}$-ray diffraction, and FT-IR spectra. It is found that the Ni-Fe LDH have a flake-like morphology, the mean diameter of $\mathrm{Pt}$ nanoparticles in $\mathrm{Pt} / \mathrm{Ni}-\mathrm{Fe} \mathrm{LDH}$ and $\mathrm{Pt} / \mathrm{IL} / \mathrm{Ni}-\mathrm{Fe} \mathrm{LDH}$ electrocatalyst is $4.36 \mathrm{~nm}$ and $3.09 \mathrm{~nm}$, smaller than that in pure Pt black and commercial Pt/C electrocatalyst $(8.70 \mathrm{~nm}$ and $6.48 \mathrm{~nm})$.
\end{abstract}

Keywords: Electrocatalyst, Methanol oxidation, Ionic liquid, Pt/Ni-Fe layered double hydroxide

\section{FULL TEXT}

(C) 2019 The Authors. Published by ESG (www.electrochemsci.org). This article is an open access article distributed under the terms and conditions of the Creative Commons Attribution license (http://creativecommons.org/licenses/by/4.0/). 